\title{
Texture Classification based on First Order Local Ternary Direction Patterns
}

\author{
M. Srinivasa Rao \\ Associate Professor, Dept of CSE, Sri Vasavi Institute of Engineering \& Technology, pedana, Andhrapradesh, India \\ Email: srinu.mekala@gmail.com \\ V.Vijaya Kumar \\ Professor, Dean and Director for CACR, Anurag Group of Institutions (Autonomous), Hyderabad, Telangana, India \\ Email: vakula_vijay@yahoo.com \\ Mhm Krishna Prasad \\ Professor of the CSE, University College of Engineering, Kakinada (Autonomous), JNTUK, Andhra Pradesh, India \\ Email: krishnaprasad.mhm@gmail.com
}

\begin{abstract}
The local binary pattern (LBP) and local ternary pattern (LTP) are basically gray scale invariant, and they encode the binary/ ternary relationship between the neighboring pixels and central pixel based on their grey level differences and derives a unique code. These traditional local patterns ignore the directional information. The proposed method encodes the relationship between the central pixel and two of its neighboring pixel located in different angles $(\alpha, \beta)$ with different directions. To estimate the directional patterns, the present paper derived variation in local direction patterns in between the two derivates of first order and derived a unique First order -Local Direction variation pattern (FO-LDVP) code. The FO-LDVP evaluated the possible direction variation pattern for central pixel by measuring the first order derivate relationship among the horizontal and vertical neighbors $\left(0^{\circ} \mathrm{Vs} .90^{\circ} ; 90^{\circ} \mathrm{Vs}\right.$. $180^{\circ} ; 180^{\circ}$ Vs. $270^{\circ} ; 270^{\circ}$ Vs. $0^{\circ}$ ) and derived a unique code. The performance of the proposed method is compared with LBP, LTP, LBPv, TS and CDTM using the benchmark texture databases viz. Brodtaz and MIT VisTex. The performance analysis shows the efficiency of the proposed method over the existing methods.
\end{abstract}

Index Terms - Local binary pattern (LBP), local ternary pattern (LTP), grey scale variance, derivative, direction

\section{INTRODUCTION}

Texture characteristics provide important information for classification and retrieval of images. The challenging issues of texture descriptors are how to deal efficiently with variations in illumination, scale, orientation, etc... The constraints are the dimensionality and computational complexity. Texture is a fundamental feature of the appearance of object surfaces and is extensively used in object surface recognition and description. Texture is considered as a "neighborhood property". One of the important and crucial tasks of image, video processing and pattern recognition is texture classification and it consequently requires the analysis of patterns in local pixel neighborhoods. The major challenges of texture classification are how to extract texture features that are insensitive to illumination changes, noise and rotationinvariant. An extensive work is carried out and a broad range of texture classification methods are proposed based on statistical properties of texture. This begins with the co-occurrence matrix method [1], which is one of the pioneering and still the bench mark method for texture classification. Later, a number of texture classification methods that were rotation- invariant are introduced. The well-known rotational invariant texture classification methods are based on: a circular autoregressive model [2] Markov random fields [3]-[5] and filter bank responses [6]-[10]. Recently texture classification methods based on local features gained lot of recognition in the research community, due to their ability in capturing more discriminative information of textures in local patterns for their respective applications. It started by the revolutionary approach derived by Ojala et al.to derive texture features by quantizing the local pixel values of a neighborhood in to two values and named it as local binary patterns (LBPs) [11,12]. Later several authors [13-19] carried out abundant work and derived efficient methods to further extend the benefits of LBP in various applications. The Binary features [12, 13, 15, 20, 21, 22] gained reputation and recognition due to their efficient design, computational simplicity and good performance. The LBP based methods are utilized in various applications, such as face recognition [23, 24,25], texture classification [26], facial expression recognition [27], age classification [28,29], image retrieval [30, 31, 32, 33], etc...The dimensionality of these descriptors grows exponentially by increasing the window size and number of neighbors considered resulting in, both, storage and computation complexity. After studying in detail on the methods based on LBP, we found one of the disadvantages of these methods is they completely ignored in measuring the directional features of texture information. The present paper derived a novel approach by measuring first order directional pattern measures 
between two neighboring pixels (yi, yj) located at an angle $\alpha o$ and $\beta o$ around the central pixel. Many variants of LBP are proposed in the literature to improve the efficiency in representing the texture features: a variant of LBP used magnitude also as a descriptor[14],other variant discarded the some patterns with few occurrences[15], many researchers combined LBP with GLCM features and also with textons [16,36,37,231,9114]. The present also derived GLCM and its features on the proposed first order derivative patterns to increase the texture classification rate. Some researchers improved the threshold mechanism of LBP [34] to sustain against noise. The other approaches used vector quantization $[38,39,40]$ to reduce the dimensionality.

A good classification results are obtained by LBP and many of LBP-like methods on different representative texture databases, they still have limitations. Firstly, the LBP and the related methods use scalar quantization in deriving binary patterns for neighborhood pixels, which is coarse. Secondly, they ignore completely the local texture derivative information. This paper addresses the above problems of LBP based methods by deriving a more efficient method called First Order Local Derivative Variation Pattern (FO-LDVP), which evaluates the relationship between the two first order derivatives along horizontal and vertical directions and represented this using a ternary pattern for deriving a new and more comprehensive local descriptor. There will be four such relationships (0o Vs.90o; 90o Vs. 180o ; 180o Vs.270o ; 270o Vs. 0o) around a central pixel of a circular or rectangle neighborhood.

The rest of the paper is organized as follows. The LBP and LTP are briefly introduced with their limitations in section two. The section three gives a detailed description about the proposed method FO-LDVP while section four presents results and discussions. Finally the conclusions are drawn in section five.

\section{MAIN CONTRIBUTION}

\section{A. Local Binary Pattern (LBP)}

LBP is a useful tool to model texture images and it is defined as a gray scale invariant texture measure. To invariance feature is achieved in LBP by deriving the gray level value difference from the central pixel and all other gray levels of the neighboring pixels. By using a sign function on this difference the local neighborhood is quantized in to binary pattern. This binary pattern is coded as 8-bit word on $3 \times 3$ neighborhoods with 8neighboring pixels. This binary word is multiplied by binary weights and thus deriving to represent the 8different-jont histograms as a 1-D histogram with 256 bins. This results a LBP code for each central pixel of a 3 x 3 neighborhood ranging from 0 to 255. This operation can be viewed as applying a bank of oriented difference filters [44]. The range of LBP codes grow large if the size of the neighborhood increases. The Fig.1 and Fig.2 show the formation of LBP code. The LBP code is evaluated by the Eq. (1) and (2).

$$
\begin{array}{|l|l|l|}
\hline \mathrm{Y}_{1} & \mathrm{Y}_{2} & \mathrm{Y}_{3} \\
\hline \mathrm{Y}_{8} & \mathrm{Y}_{\mathrm{C}} & \mathrm{Y}_{4} \\
\hline \mathrm{Y}_{7} & \mathrm{Y}_{6} & \mathrm{Y}_{5} \\
\hline
\end{array}
$$

Fig.1. A 3x3 neighborhood.

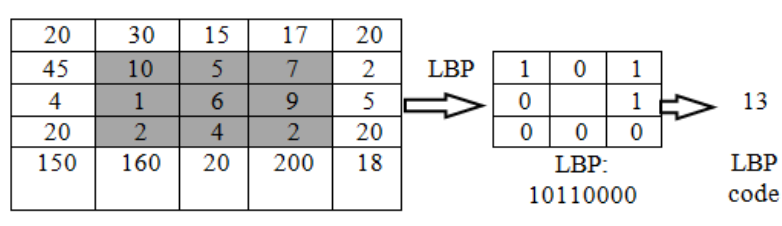

Fig.2. An example of calculating LBP code.

$$
\begin{gathered}
L B P_{P, R}=\sum_{p=1}^{P} 2^{p-1} * f\left(Y_{p}-Y_{c}\right) \mathrm{p}=1,2 \ldots 8 \\
f(Y)=\left\{\begin{array}{c}
1 Y \geq 0 \\
0 \text { else }
\end{array}\right.
\end{gathered}
$$

In Eq. (1), $P$ is number of neighboring pixels, $R$ is radius, $Y p$ and $Y_{c}$ are the gray level value of neighboring and central pixel.

\section{B. Local Ternary Pattern (LTP)}

The concept of LBP is extended to three values code by Tan and Trigs [45] by adding a threshold factor $t_{f}$ to the $Y_{c}$. The pattern indicator $f(Y)$ is quantized to zero, +1 and -1 based on Eq.(3)

$$
\begin{gathered}
\operatorname{LTP}_{P, R}=\sum_{p=1}^{P} 3^{p-1} * f\left(Y_{p}-Y_{c}\right) \\
f(Y)=\left\{\begin{array}{c}
+1 Y_{p} \geq Y_{c}+t_{f} \\
0\left|Y_{p}-Y_{c}<t_{f}\right| \\
-1 Y_{p} \leq Y_{c}-t_{f}
\end{array}\right.
\end{gathered}
$$

\section{First Order-Local derivative variation Pattern (FO-} LDVP)

The LBP and LTP are simple to implement, resistance to lighting changes and efficient ability to encode fine details. That's why there are popular and widespread local descriptors among the researchers in computer vision and pattern recognition. The common problems of these (LBP and LTP based methods) are they all ignore the spatial distribution feature of texture in images e.g., the varying texture features in different directions. This will cause some loss of significant and useful texture information and by which the classification performance will be degraded. In conclusion, LBP and LTP derives the texture information based on grey level variation pattern of pixels, which is coded using only two directions (positive direction or negative direction) and they completely ignore the information regarding the direction of local features. This observation made us to propose the computation of gray-level derivative variation patterns on different directions and construct the spatial distribution pattern. The present paper evaluated the possible ternary directions for central pixel by measuring the first order derivates relationship among 
the horizontal and vertical neighbors (i.e. $0^{\circ} \mathrm{Vs} .90^{\circ} ; 90^{\circ}$ Vs. $180^{\circ} ; 180^{\circ}$ Vs. $270^{\circ} ; 270^{\circ}$ Vs. $360^{\circ}$ ). From this, the proposed FO-LDVP derived a number for the $3 \times 3$ neighborhood ranging from 0 to 80 and the detailed description is given below.

The present paper derived four first order derivatives along $0^{\circ}, 90^{\circ}, 180^{\circ}$ and $270^{\circ}$ around the central pixel of the neighborhood and they can be written as

$$
\begin{gathered}
f_{0^{o}}^{I}\left(Y_{c}\right)=f\left(Y_{4}\right)-f\left(Y_{0}\right) \\
f_{90^{\circ}}^{I}\left(Y_{c}\right)=f\left(Y_{2}\right)-f\left(Y_{0}\right) \\
f_{180^{o}}^{I}\left(Y_{c}\right)=f\left(Y_{8}\right)-f\left(Y_{0}\right) \\
f_{270^{o}}^{I}(c)=f\left(Y_{6}\right)-f\left(Y_{0}\right)
\end{gathered}
$$

The present paper derived local direction patterns in between the above two derivates of first order and named it as "First Order - Local derivative variation Pattern (FO-LDVP)". The present paper derived four different First Order (FO) Local Derivative variation Patterns between two neighboring pixels located with angle of $\alpha^{\circ}$ and $\beta^{\circ}$ around the central pixel. The four FO-local derivative patterns are defined as i. $0^{\circ} \mathrm{Vs} .90^{\circ}\left(D_{1}^{I}\left(Y_{c}\right)\right)$ ii. $90^{\circ}$ Vs. $180^{\circ}\left(D_{2}^{I}\left(Y_{c}\right)\right)$ iii. $180^{\circ}$ Vs. $270^{\circ}\left(D_{3}^{I}\left(Y_{c}\right)\right)$ iv. $270^{\circ}$ Vs. $0^{\circ}\left(D_{4}^{I}\left(Y_{c}\right)\right)$.

The proposed FO-LDVP assigns a ternary pattern $(0,1$, 2) by comparing the two neighboring pixel gray values $\left(\mathrm{y}_{\mathrm{i}}, \mathrm{y}_{\mathrm{j}}\right)$ located at angle $\alpha^{\mathrm{o}}$ and $\beta^{\mathrm{o}}$ respectively with the central pixel (two local derivatives). These ternary patterns represent the derivative direction patterns of the central pixel along with its two neighboring pixels $\left(y_{i}\right.$, $\mathrm{y}_{\mathrm{j}}$ ). The present paper transforms these derivative variation patterns into a decimal number to represent the direction of the structural pattern. This decimal number is denoted as FO-LDVP code. The present paper defined the possible direction of the central pixel in each of the above four first-order derivative directions with a ternary value $(0,1,2)$ as given in the Eq. (5). The Eq. (6) gives the FO-LDVP code.

for $\mathrm{d}=\{1,2,3,4\}$

$$
\begin{gathered}
D_{d}^{I}\left(Y_{c}\right)=\left\{\begin{array}{cc}
2 & f_{\alpha^{0}}^{I}\left(Y_{c}\right) \geq 0 \text { and } f_{\beta^{0}}^{I}\left(Y_{c}\right) \geq 0 \\
1 & f_{\alpha^{0}}^{I}\left(Y_{c}\right)<0 \text { and } f_{\beta^{0}}^{I}\left(Y_{c}\right)>0 \\
& \text { or } \\
& f_{\alpha^{0}}^{I}\left(Y_{c}\right)>0 \text { and } f_{\beta^{0}}^{I}\left(Y_{c}\right)<0 \\
0 & f_{\alpha^{0}}^{I}\left(Y_{c}\right)<0 \text { and } f_{\beta^{0}}^{I}\left(Y_{c}\right)<0
\end{array}\right. \\
F O-L D V P=\sum_{D=1}^{4} 3^{D-1} * D_{d}^{I}\left(Y_{c}\right)
\end{gathered}
$$

The Eq. (5) gives three possible derivative direction values for central pixel in each case. This derives four ternary pattern values on a 3x3 local neighborhood and by multiplying them with ternary weights and later concatenating them, will yield FO-LDVP code as given in Eq. (6). The FO-LDVP code ranges from 0 to 80. The Fig.3 illustrates FO-LDVP around the central pixel $\mathrm{Y}_{\mathrm{c}}$ considered in this paper.

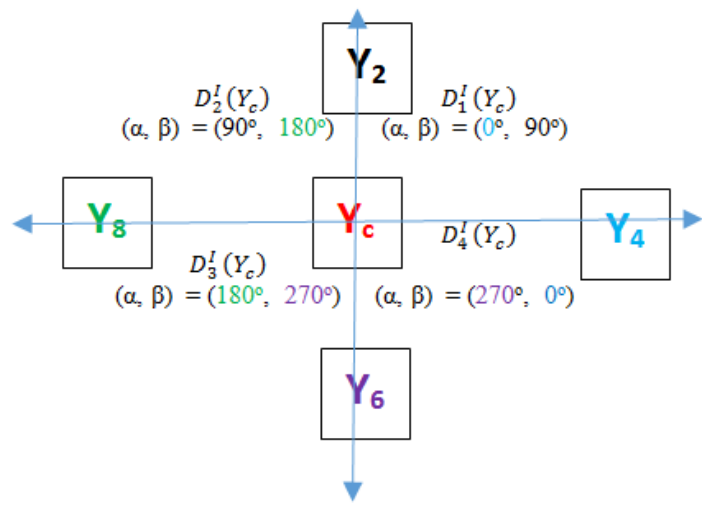

Fig.3. Representation of four FO-LDVP.

The central pixel $Y_{c}$ is marked with red color. The pixel that forms an angle $0^{\circ}, 90^{\circ}, 180^{\circ}$ and $270^{\circ}$ are shown with blue $\left(\mathrm{Y}_{4}\right)$, black $\left(\mathrm{Y}_{2}\right)$, green $\left(\mathrm{Y}_{8}\right)$ and purple $\left(\mathrm{Y}_{6}\right)$ colors respectively.

An example of FO-LDVP along the four different directions with respect to central pixel is given in Fig.4. When we apply the Eq. (6) for first combination i.e. $\alpha=$ $0^{\circ}$ and $\beta=90^{\circ}\left(Y_{2}<-Y_{c^{-}}>Y_{4}\right)$, it has resulted a ternary value 2 . In the same way the other combinations $\alpha=90^{\circ}$ and $\beta=180^{\circ}\left(\mathrm{Y}_{8}<-\mathrm{Y}_{\mathrm{c}^{-}}>\mathrm{Y}_{2}\right)$ resulted a ternary value 1 and other two ternary values for the combinations $\alpha=180^{\circ}$ and $\beta=270^{\circ}$ and $\alpha=270^{\circ}$ and $\beta=0^{\circ}$ are computed and shown in Fig.4. This results a FO-LDVP code 32 for the above Fig.4. The central pixel is replaced by this code.

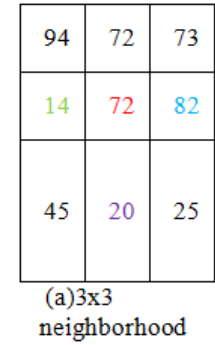

Fig.4. Generation of first order local derivative variation pattern (FOLDVP ) code.

\section{Gray Level Co-occurrence Matrix (GLCM)}

The present paper derived GLCM and other features on the FO-LDVP matrix image for efficient classification. The grey level range of FO-LDVP matrix ranges from 0 to 80 . Therefore the size of the GLCM on FO-LDVP will be $81 \times 81$. The GLCM is one of the popular and bench mark method and it was derived by Haralick et al. [1]. GLCM is a second order statistical method. Construction of GLCM is based on the spatial relationship between gray tones in an image [46] and with this property it is able to characterize texture images very efficiently. The GLCM computation is given below: Initially the original texture image I quantized with reduced number of gray levels, from 0 to $g-1$. Then, GLCM is computed from the quantized image by scanning the intensity of each pixel and its neighbor, defined by displacement $\mathrm{d}$ and angle $\varnothing$. A displacement, $\mathrm{d}$ could take a value of $1,2,3 \ldots \mathrm{n}$ 
whereas an angle, $\varnothing$ is limited $0^{\circ}, 45^{\circ}, 90^{\circ}$ and $135^{\circ}$. The following Fig.5 illustrates the formation of GLCM with different angles $\left(0^{\circ}, 45^{\circ}, 90^{\circ}\right.$ and $\left.135^{\circ}\right)$ from a given window.

\begin{tabular}{|l|l|l|}
\hline 3 & 3 & 3 \\
\hline 1 & 3 & 3 \\
\hline 1 & 3 & 2 \\
\hline
\end{tabular}

\begin{tabular}{|c|c|c|c|}
\hline $0^{\circ}$ & 1 & 2 & 3 \\
\hline 1 & 0 & 0 & 2 \\
\hline 2 & 0 & 0 & 0 \\
\hline 3 & 0 & 1 & 3 \\
\hline
\end{tabular}

\begin{tabular}{|c|c|c|c|}
\hline $45^{\circ}$ & 1 & 2 & 3 \\
\hline 1 & 0 & 0 & 2 \\
\hline 2 & 0 & 0 & 0 \\
\hline 3 & 0 & 0 & 2 \\
\hline
\end{tabular}

\begin{tabular}{|c|c|c|c|}
\hline $90^{\circ}$ & 1 & 2 & 3 \\
\hline 1 & 1 & 0 & 1 \\
\hline 2 & 0 & 0 & 1 \\
\hline 3 & 0 & 0 & 3 \\
\hline
\end{tabular}

\begin{tabular}{|c|c|c|c|}
\hline $135^{\circ}$ & 1 & 2 & 3 \\
\hline 1 & 0 & 0 & 0 \\
\hline 2 & 0 & 0 & 1 \\
\hline 3 & 0 & 0 & 2 \\
\hline
\end{tabular}

Fig.5. An example of GLCM formation. (a): The sample window. (b) (c) (d) and (e ) are $0^{\circ}, 45^{\circ}, 90^{\circ}$ and $135^{\circ}$ degrees GLCM matrix of (a) respectively with a distance $\mathrm{d}=1$;

The present paper initially constructed GLCM on FOLDVP with $\mathrm{d}=1,2,3$ and 4 and with $0^{\circ}, 45^{\circ}, 90^{\circ}$ and $135^{\circ}$ degrees. The four GLCM features i) Contrast, ii) Correlation, iii) Energy iv) homogeneity with $0^{\circ}, 45^{\circ}$, $90^{\circ}$ and $135^{\circ}$ are evaluated on the referred database images and average of these are considered for texture classification. The four GLCM features are given below:

1. Contrast :

Contrast $=\sum_{n=0}^{M-1} n^{2}\left\{\sum_{i=1}^{M} \sum_{j=1}^{N} X(i, j)\right\},|i-j|=n$

2. Correlation :

$$
\text { Correlation }=\sum_{i=0}^{M-1} \sum_{j=0}^{N-1} \frac{\{i X j\} X X(i, j)-\left\{\mu_{x} X \mu_{y}\right\}}{\sigma_{x} X \sigma_{y}}
$$

3. Energy :

$$
\text { Energy: } \sum_{i, j} X(i, j)^{2}
$$

4. Homogeniety, Angular Second Moment (ASM):

$$
\mathrm{ASM}=\sum_{i=0}^{G-1} \sum_{j=0}^{G-1}\{X(i, j)\}^{2}
$$

Further the present paper evaluated the following nine features on GLCM of FO-LDVP image matrix: mean, standard deviation, entropy, root mean square error (RMS), variance, smoothness, kurtosis, skewness and inverse difference movement (IDM) with different orientations $\left(0^{\circ}, 45^{\circ}, 90^{\circ}\right.$ and $\left.135^{\circ}\right)$ and average of this is considered for classification. They are given below.

5. Mean

$$
\text { Mean }=\frac{1}{n} \sum_{i=0}^{M-1} \sum_{j=0}^{N-1} X(i, j)
$$

6. Standard deviation
Standard deviation $=\frac{1}{n} \sum_{i=0}^{M-1} \sum_{j=0}^{N-1} X(i, j)-\mu$

7. Entropy:

$$
\text { Entropy }=-\sum_{i=0}^{G-1} \sum_{j=0}^{G-1} X(i, j) * \log (X(i, j))
$$

8. Root mean square (RMS)

$$
\mathrm{RMS}=\sqrt{\frac{1}{n}}\left(x_{1}^{2}+x_{2}^{2}+x_{3}^{2}+\cdots .+x_{n}^{2}\right)
$$

9. Skewness : The skewness of a distribution is defined as

$$
S=\frac{E(X-\mu)^{3}}{\sigma^{3}}
$$

10. Variance

$$
\text { Variance }=\frac{\sum(X-\mu)^{2}}{N}
$$

11. Inverse Difference Moment (IDM): It is the local homogeneity. It is high when local gray level is uniform and inverse GLCM is high.

$$
I D M=\frac{\sum_{i=0}^{M-1} \sum_{0}^{N-1} X_{i j}}{1+(i-j)^{2}}
$$

Where $\mathrm{X}$ is image with size $\mathrm{MxN}$

12. Smoothness

$$
\text { Smoothness }=1-\frac{1}{1+\sum_{i=0}^{M-1} \sum_{j=0}^{N-1} X(i, j)}
$$

where $\mathrm{X}$ is image

13. Kurtosis: The kurtosis of a distribution is defined as

$$
k=\frac{E(X-\mu)^{4}}{\sigma^{4}}
$$

Where the following are defined on GLCM (derived from FO-LDVP ) : $\mathrm{X}$ is GLCM , $\mathrm{n}$ is number of elements, $\mathrm{X}(\mathrm{i}, \mathrm{j})$ represent pixel element, $\mathrm{x}_{1}, \mathrm{x}_{2} \ldots \mathrm{x}_{\mathrm{n}}$ are intensity values, $\mu$ is the mean, $\sigma$ is the standard deviation and $E(t)$ represents the expected value of the quantity $t$.

The machine learning classifiers are used for classification purpose. Classification is done in two different ways one is only by considering the four GLCM features on FO-LTDP and the other is by integrating GLCM features on FO-LTDP with the other nine futures.

\section{EXPERIMENTAL RESULTS}

To examine the efficacy of the proposed method, we carried out a series of experiments on two representative texture databases: Brodatz [47] and MIT VisTex [48]. The advantage of these different texture databases, they 
are constructed under different viewpoints, conditions of illumination, and scale. The present paper classified the textures in two ways with different displacements on various machine learning classifiers i) by using the only four texture features of Haralick ii) by integrating all the 13 features that are derived on the proposed GLCM on FO-LDVP as given in section $\mathrm{C}$. The present paper measured texture classification rate using the following machine learning classifiers 1)Multi perceptron, 2) Naïvebayes, 3)J48 and 4) lbk. The proposed GLCM on FO-LTDP is compared with other representative LBP schemes, such as the LBP[12], the LTP [45], the CDTM scheme [49]] and TS [50] approaches.

\section{A. Experimental Results on Brodatz Database}

We selected 10 homogeneous texture image classes from the Brodatz database, as shown in Fig. 6 (Granite, Bark, Wood, Marble, Stone, Gravel, Wall, Brick, Pigskin and Grass). In each class, we have considered 6 texture images. The texture images are of size 512 x 512 pixels. Then, each image is divided in to 64, non-overlapped patches of size $64 \times 64$. In this way, we generated a dataset containing $3840(10 \times 6 \times 64)$ texture images, and with each class or category containing 384 images. We have considered 3-texture images $(3 \times 64=192)$ in each category as training and other remaining three images (192 sub images) as test images, i.e. $50 \%$ of images in each class are considered as test images. Test images are not part of database images.
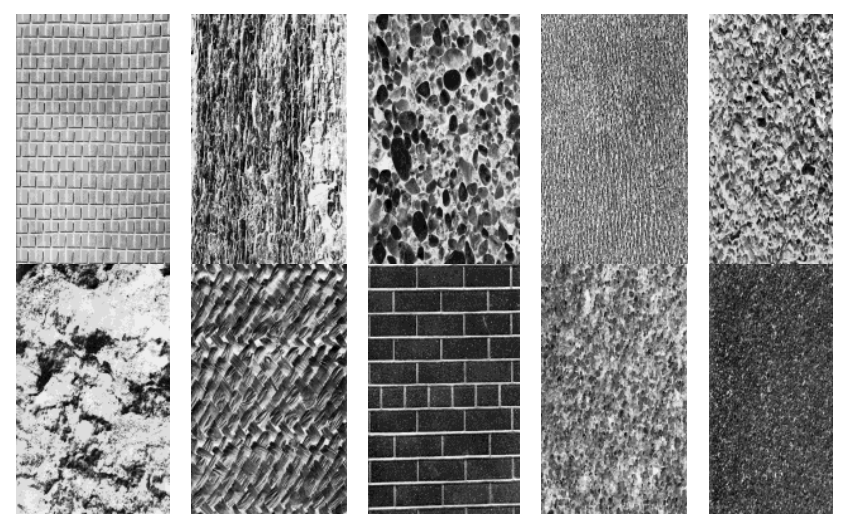

Fig.6. Brodtaz texture database.

\section{B. Experimental Results on MIT VisTex Database}

The MIT VisTex database is an alternative collection for Brodatz texture library and images of this are representative of real world conditions. These images do not conform to rigid frontal plane perspectives and studio lighting conditions. The MIT VisTex also comprises examples of many non-traditional textures. The present paper used 10 classes of MIT VisTex album texture images with $128 \times 128$ pixels and in each class 6 images are selected randomly. The classes of MIT VisTex images considered are (Bark, Brick, Grass, Wall, Stone, Gravel, Water, Granite, Wood and Marble) and shown in Fig. 7. Each texture image is sub divided in to sub images of size $64 \times 64$. This results a total of 4 sub images for each image and a total of 240 (10x 6x 4) image databases.
Out of this $50 \%$ of images are considered for training data set in each class.
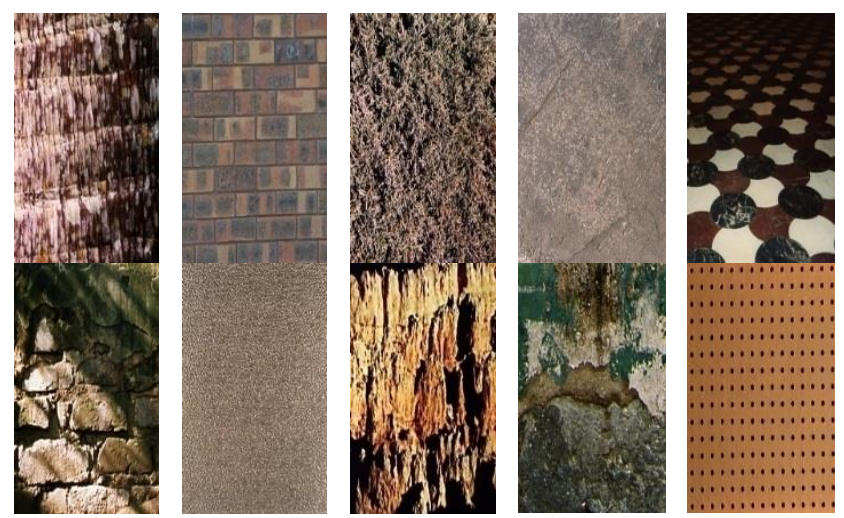

Fig.7. MIT VisTex texture database.

The table 1 and 2 shows the average classification rate by the proposed FO-LDVP method with four GLCM features and integrated features (13 features) respectively, with varying distances by using machine learning classifiers on both considered data bases. The graphs of Fig. 8 and Fig. 9 shows the classification rate of the proposed method. The graphs clearly indicates the proposed integrated GLCM features of FO-LDVP have shown high classification rate for $\mathrm{d}=3$ and by using Ibk classifier. The Fig.10 compares the performance of the proposed FO-LDVP method for $\mathrm{d}=3$ using 4-GLCM and integrated features and integrated features on proposed method shown high classification rate on all classifiers.

Table 1. Average classification rate of the proposed FO-LDVP using GLCM features.

\begin{tabular}{|l|l|c|c|c|c|}
\hline \multirow{2}{*}{$\begin{array}{l}\text { Texture } \\
\text { database }\end{array}$} & \multirow{2}{*}{ Classifiers } & \multicolumn{4}{|c|}{ Classification rate (\%) } \\
\cline { 3 - 6 } & & \multicolumn{3}{|c|}{ Distance value d on GLCM } \\
\cline { 3 - 6 } & & $\mathrm{d}=1$ & $\mathrm{~d}=2$ & $\mathrm{~d}=3$ & $\mathrm{~d}=4$ \\
\hline Brodtaz & $\begin{array}{l}\text { Multilayer } \\
\text { Perceptron }\end{array}$ & 83.69 & 84.58 & 85.04 & 84.48 \\
\cline { 2 - 6 } & Ibk & 84.74 & 88.45 & 90.47 & 89.78 \\
\cline { 2 - 6 } & J48 & 85.36 & 87.36 & 89.69 & 87.82 \\
\cline { 2 - 6 } & Logistic & 74.85 & 78.68 & 79.35 & 77.85 \\
\hline \multirow{3}{*}{$\begin{array}{l}\text { MIT } \\
\text { VisTex }\end{array}$} & $\begin{array}{l}\text { Multilayer } \\
\text { Perceptron }\end{array}$ & 80.49 & 81.42 & 82.48 & 82.01 \\
\cline { 2 - 6 } & Ibk & 83.14 & 85.41 & 87.48 & 86.48 \\
\cline { 2 - 6 } & J48 & 79.89 & 80.76 & 82.75 & 81.45 \\
\cline { 2 - 6 } & Logistic & 69.48 & 70.88 & 75.74 & 74.87 \\
\hline
\end{tabular}

Table 2. Average classification rate of the proposed FO-LTD ${ }_{\text {ir }} \mathrm{P}$ using all 13 features.

\begin{tabular}{|l|l|c|c|c|c|}
\hline \multirow{2}{*}{$\begin{array}{l}\text { Texture } \\
\text { database }\end{array}$} & \multirow{2}{*}{ Classifiers } & \multicolumn{4}{|c|}{ Classification rate (\%) } \\
\cline { 3 - 6 } & & \multicolumn{4}{|c|}{ Distance value d on GLCM } \\
\cline { 3 - 6 } & $\begin{array}{l}\text { Multilayer } \\
\text { Perceptron }\end{array}$ & 92.15 & 95.13 & 97.22 & 96.45 \\
\cline { 2 - 6 } & Ibk & 93.25 & 96.49 & 98.48 & 96.68 \\
\cline { 2 - 6 } & J48 & 89.78 & 91.45 & 94.91 & 93.89 \\
\cline { 2 - 6 } & Logistic & 94.15 & 96.59 & 97.37 & 95.78 \\
\hline \multirow{4}{*}{$\begin{array}{l}\text { MIT } \\
\text { VisTex }\end{array}$} & $\begin{array}{l}\text { Multilayer } \\
\text { Perceptron }\end{array}$ & 84.68 & 87.68 & 91.2 & 90.24 \\
\cline { 2 - 6 } & Ibk & 74.85 & 80.41 & 89.95 & 86.36 \\
\cline { 2 - 6 } & J48 & 72.65 & 79.68 & 86.68 & 84.69 \\
\cline { 2 - 6 } & Logistic & 80.16 & 84.41 & 89.67 & 87.16 \\
\hline
\end{tabular}




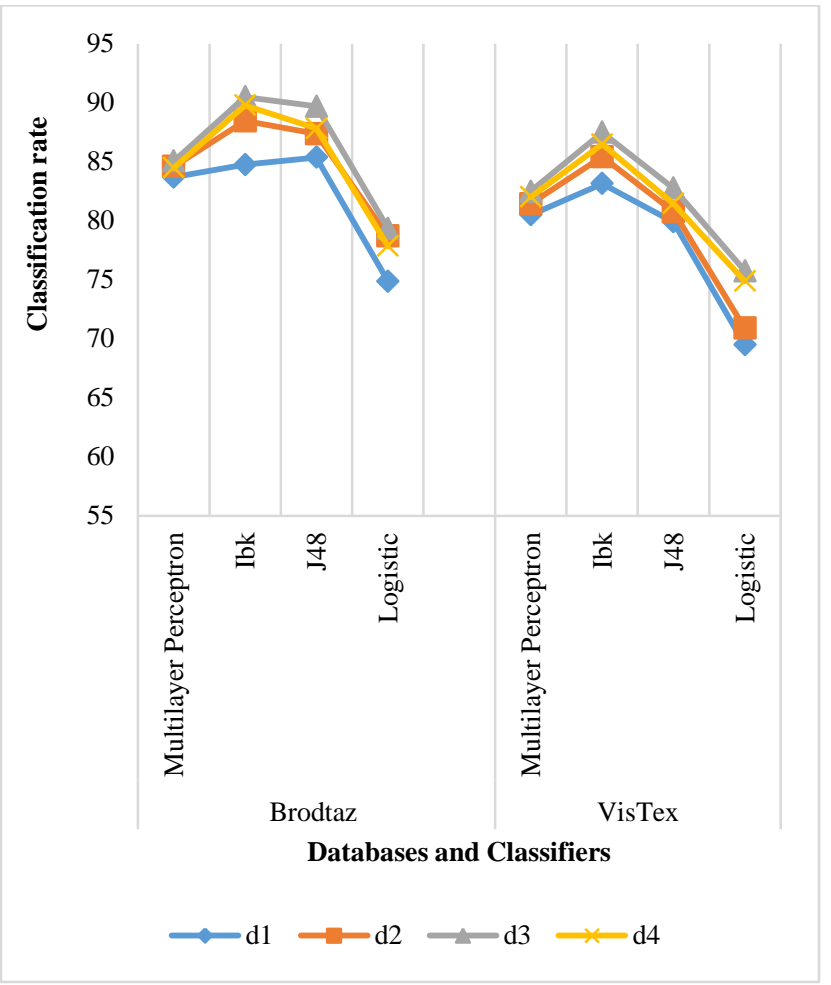

Fig.8. Classification graph using four GLCM features for the proposed method using various classifiers for $\mathrm{d}=1,2,3$ and 4 .

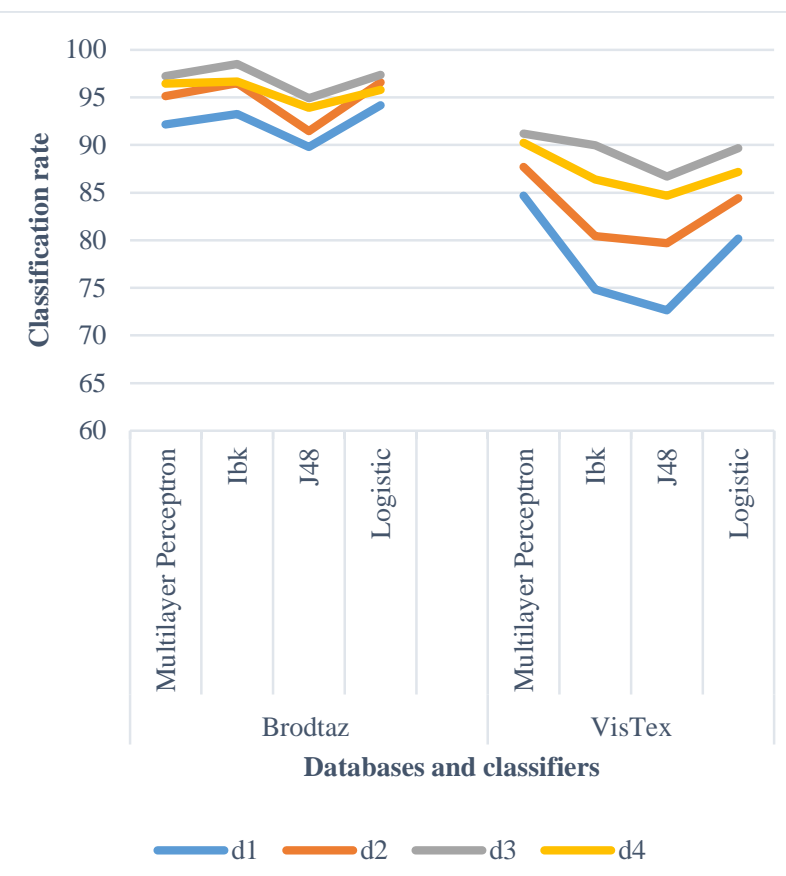

Fig.9. Classification graph for the proposed method with integrated features (all 13 features)

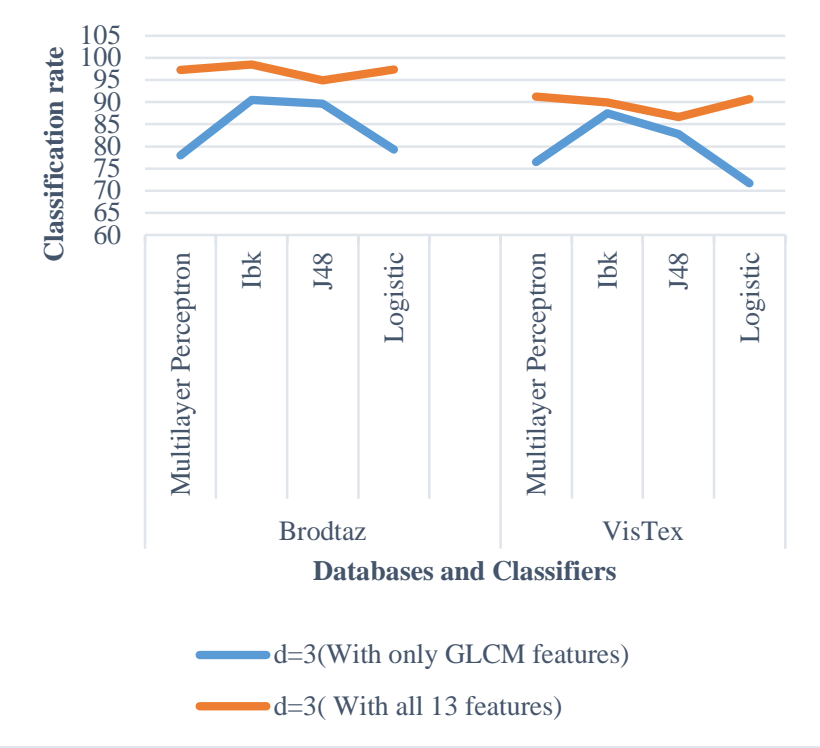

Fig.10. Classification graph for the proposed method with integrated features (all 13 features) and with four GLCM features for $\mathrm{d}=3$.

The Table 3 and 4 shows the classification results over the Brodatz and VisiTex database of the proposed and other methods, which are based on LBP model.

Table 3. Classification rate on Brodtaz database.

\begin{tabular}{|l|c|}
\hline Methods & Classification rate (\%) \\
\hline LBP & 40.58 \\
\hline LTP & 57.85 \\
\hline LBP with variance & 66.61 \\
\hline TS[50] & 38.98 \\
\hline CDTM[49] & 82.73 \\
\hline $\begin{array}{l}\text { Proposed FO-LTDP with only 4 } \\
\text { features of GLCM for d=3 }\end{array}$ & 91.62 \\
\hline $\begin{array}{l}\text { Proposed FO-LTDP with integrated } \\
\text { features (13 features as Specified in } \\
\text { section C)d=3 }\end{array}$ & 98.69 \\
\hline
\end{tabular}

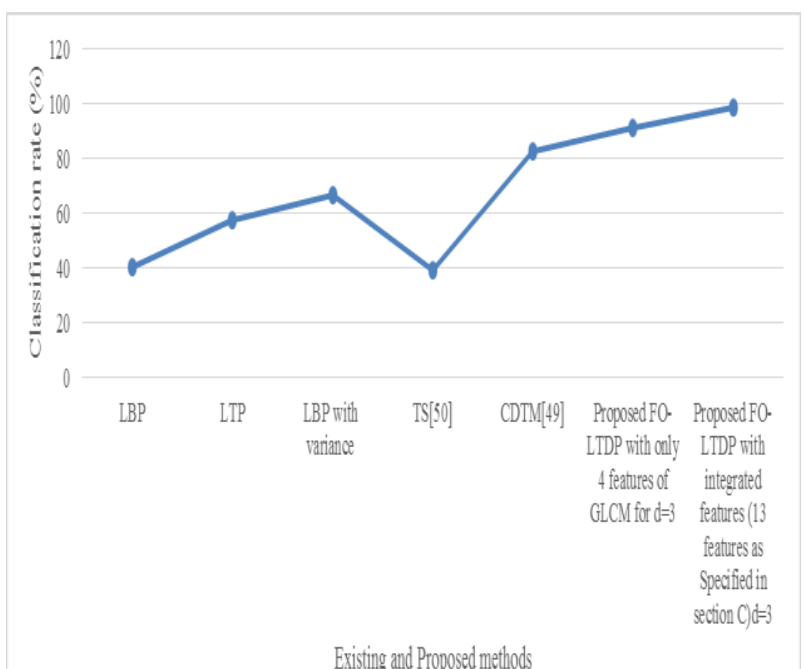

Fig.11. Comparison graph of proposed and existing methods on Brodtaz database. 
Table 4. Classification rate on MIT VisTex database.

\begin{tabular}{|l|l|}
\hline Methods & Classification rate (\%) \\
\hline LBP & 39.95 \\
\hline LTP & 52.36 \\
\hline LBP with variance & 64.21 \\
\hline TS[50] & 38.21 \\
\hline CDTM[49] & 80.45 \\
\hline $\begin{array}{l}\text { Proposed FO-LTD }{ }_{\text {ir }} \text { P with only 4 } \\
\text { features of GLCM for d=3 }\end{array}$ & 89.68 \\
\hline $\begin{array}{l}\text { Proposed FO-LTD } \\
\text { features (13 features as specified in } \\
\text { section C) for d=3 }\end{array}$ & 92.74 \\
\hline
\end{tabular}

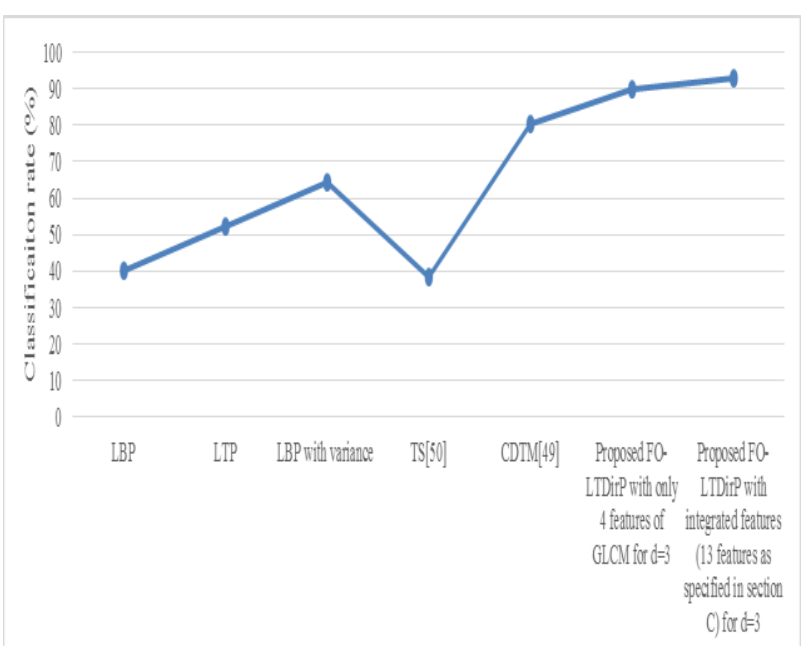

Exising and Proposed lethols

Fig.12. Comparison graph of proposed and existing methods on MIT VisTex database.

Table 3 shows the experimental results of all the listed methods. The $\mathrm{LBPv}$ achieves better results than LBP because it captures the magnitude information of the difference vector; however the coarse quantization over the variance limits its performances.

The results are presented in Table 3. As we can see from there, reducing the dimensionality of our feature vectors decreases the classification accuracy but the performance drop is relatively small. In particular, the achieved results still outperform those obtained for conventional LBP and LTP based approaches and show that we are able to derive an improved texture descriptor that is as compact and provides improved texture classification with reduced dimensionality. The best results are obtained for $\mathrm{d}=3$ and on lbk classifier. Our experimental results, obtained from two representative databases (MIT Visitex and Brodatz), clearly show that FO-LDVP has higher texture classification accuracy than LBP and LBP-like methods.

\section{CONCLUSIONS}

In this paper, we explored two disadvantages of LBP and LBP-like methods and found that they have ignored completely the direction responses around the central pixel. In this paper we have proposed a novel approach referred as FO-LDVP for texture image classification. The FO-LDVP used first order horizontal and vertical pixels relationship with central pixel and derived a ternary direction pattern. This process is continued for both horizontal pixels and vertical pixels around the central pixel i.e. $0^{\circ}$ Vs. $90^{\circ}, 90^{\circ}$ Vs. $180^{\circ}, 180^{\circ}$ Vs. $270^{\circ}$ and $270^{\circ} \mathrm{Vs}$. $0^{\circ}$. The performance of the proposed method is compared with the LBP, LTP, LBPv, TS and CDTM methods. The range of FO-LDVP greatly reduced to 0 to 80 when compared to 0 to 255 (in case of LBP) and 0 to 3561 (in case of LTP). Finally, we derived the GLCM on FO-LDVP and this will have a dimension of $80 \times 80$ and it also reduced the dimensionality of GLCM from $255 \times 255$ and $3561 \times 3561$ in case of LBP and LTP based methods respectively. The extensive experiments results on the two referred data bases clearly demonstrate that the proposed approach outperforms LBP and LBPlike methods.

\section{REFERENCES}

[1] R. M. Haralik, K. Shanmugam, and I. Dinstein, "Texture features for image classification," IEEE Trans. Syst., Man Cybern., vol. SMC-3, no.6, pp. 610-621, Jun., 1973.

[2] R. Kashyap and A. Khotanzed, "A model-based method for rotation invarianttexture classification," IEEE Trans. Pattern Anal. Mach. Intell.,vol. PAMI-8, no. 4, pp. 472481, Apr. 1986.

[3] H. Deng and D. A. Clausi, "Gaussian MRF rotationinvariant featuresfor image classification," IEEE Trans. Image Process., vol. 26, no. 7,pp. 951-955, Jul. 2004.

[4] J. Chen and A. Kundu, "Rotation and gray scale transform invariant texture identification using wavelet decomposition and hidden Markovmodel," IEEE Trans. Pattern Anal. Mach. Intell., vol. 16, no. 2, pp.208-214, Feb. 1994.

[5] M. Varma and A. Zisserman, "A statistical approach to material classification using image patch exemplars," IEEE Trans. Pattern Anal.Mach. Intell., vol. 31, no. 11, pp. 2032-2047, Nov. 2009.

[6] Liane and J. Fan, "Texture classification by wavelet packet signature,'IEEE Trans. Pattern Anal. Mach. Intell., vol. 15, no. 11, pp.1186-1191, 1993.

[7] G. M. Haley and B. S. Manjunath, "Rotation-invariant texture classification using a complete space-frequency model," IEEE Trans. ImageProcess., vol. 8, no. 2, pp. 255-269, Feb. 1999.

[8] M. Varma and A. Zisserman, "A statistical approach to texture classificationfrom single images," Int. J. Comput. Vis., vol. 62, no. 1-2, pp.61-81, 2005.

[9] T. Randen and J. Husoy, "Filtering for texture classification: A comparativestudy," IEEE Trans. Pattern Anal. Mach. Intell., vol. 21, no. 4,pp. 291-310, Apr. 1999.

[10] L. Li, C. S. Tong, and S. K. Choy, "Texture classification using refinedhistogram," IEEE Trans. Image Process., vol. 19, no. 7, pp. 1371-1378,Jul. 2010.

[11] T. Ojala, M. Pietikäinen, and D. Harwood, “A comparative study of texture measures with classification based on feature distributions," Pattern Recognit., vol. 29, no. 1, pp. 51-59, 1996.

[12] T. Ojala, M. Pietikäinen, and T. Mäenpää, "Multiresolution gray-scale and rotation invariant texture classification with local binary patterns,"IEEE Trans. Pattern Anal. Machine Intell., vol. 24, no. 7, pp. 971987,Jul. 2002. 
[13] X. Tan and B. Triggs, "Enhanced local texture feature sets for face recognition under difficult lighting conditions," IEEE Trans. Image Process., vol. 19, no. 6, pp. 1635-1650, Jun. 2010.

[14] Z. Guo, L. Zhang, and D. Zhang, "A completed modeling of local binary pattern operator for texture classification," IEEE Trans. Image Process.,vol. 9, no. 16, pp. 16571663, Jun. 2010.

[15] S. Liao, M. W. K. Law, and A. C. S. Chung, "Dominant local binary patterns for texture classification," IEEE Trans. Image Process., vol. 18,no. 5, pp. 1107-1118, May 2009.

[16] X. Qi, R. Xiao, C.-C. Li, Y. Qiao, J. Guo, and X. Tang, "Pairwise rotation invariant co-occurrence local binary pattern," IEEE Trans. Pattern Anal.Mach. Intell., vol. 36, no. 11, pp. 2199-2213, Nov. 2014.

[17] S. Hussain and B. Triggs, "Visual recognition using local quantizedpatterns," in Proc. Eur. Conf. Comput. Vis. 2012, pp. 716-729.

[18] S. H. Lee, J. Y. Choi, Y. M. Ro, and K. N. Plataniotis, "Local color vector binary patterns from multichannel face images for face recognition,"IEEE Trans. Image Process., vol. 21, no. 4, pp. 2347-2353, Apr. 2012.

[19] Zhu and R. Wang, "Local multiple patterns based multiresolution gray-scale and rotation invariant texture classification," Inf. Sci., vol. 187,pp. 93-108, Mar. 2012.

[20] T. Ahonen, J. Matas, C. He, and M. Pietikäinen, "Rotation invariant image description with local binary pattern histogram Fourier features, "in Image Analysis. Berlin, Germany: Springer, 2009, pp. 61-70.

[21] Z. Guo, L. Zhang, and D. Zhang, "Rotation invariant texture classification using LBP variance (LBPV) with global matching," Pattern Recognit., vol. 43, no. 3, pp. 706-719, 2010.

[22] Z. Guo and D. Zhang, "A completed modeling of local binary pattern operator for texture classification," IEEE Trans. Image Process., vol. 19,no. 6, pp. 1657-1663, Jan. 2010.

[23] G S Murty ,J Sasi Kiran , V.Vijaya Kumar, "Facial expression recognition based on features derived from the distinct LBP and GLCM", International journal of image, graphics and signal processing (IJIGSP), , Vol.2, No.1, pp. 68-77,2014, ISSN: 2074-9082.

[24] V. Vijaya Kumar, K. Srinivasa Reddy, V. Venkata Krishna , "Face Recognition Using Prominent LBP Model", International Journal of Applied Engineering Research , Vol. 10, Issue 2, pp. 4373-4384, 2015, ISSN: 0973-4562

[25] Baochang Zhang, Yongsjeng Gao, Sanqiang Zhao and Jianzhuang, "Local Derivative Pattern Versus Local Binary Patter: Face Recognition with High-Order Local Pattern Descriptor", IEEE Transactions on Image Processing, Vol. 19, No.2, Feb.2010.

[26] B. Sujatha, V Vijaya Kuamr, P. Harini, "A new logical compact LBP co-occurrence matrix for texture analysis", International journal of scientific \& engineering research, Vol.3, No.2, pp.1-5, Feb-2012, ISSN 2229-5518.

[27] V. Vijaya Kumar, Gorti Satyanaraya Murty, PS V V S R Kumar, "Classification of facial expressions based on transitions derived from third order neighborhood LBP", Global journal of computer science and technology graphics \& vision (GJCST), Vol.14, N0.1, pp. 1-12, Jan2014, ISSN: 0975-4350.

[28] Gorti S Murty,V.Vijaya Kumar,A. Obulesu, "Age classification based on simple LBP transitions", International journal of computer science and engineering
(IJCSE), Vol.5, No. 10, pp.885-893, OCT-2013, ISSN: 0975-3397.

[29] V.Vijaya Kumar, P.J.S. Kumar, Pullela S V V S R Kumar, "Age classification of facial images using third order neighbourhood Local Binary Pattern", International Journal of Applied Engineering Research Volume 10, Number 15 (201105) pp 35704-35713, ISSN 0973-4562.

[30] Obulesu., A,JS Kiran, VV Kumar, "Facial image retrieval based on local and regional features", IEEE- 2015 International Conference on Applied and Theoretical Computing and Communication Technology (iCATccT), 29-31 Oct. 2015, Pp:841 - 846

[31] Ms. Shraddha D., Jumade, Prof. Sheetal S.Dhande, "An Indexing and Retrieval Method Using Local Tetra Pattern for Content -Based Image Retrieval (CBIR)", International Journal of Computer Science and Information Technologies, Vol. 6(3), 2015.

[32] Subrahmanyam Murala, R.P. Maheswari, R. Balasubramanian, "Local Tetra Pattern: A New Feature Descriptor for Content Based Image Retireval", IEEE Transaction on Image processing, Vol. 21, No:5, 2012

[33] Philominal Divya, I. Jeena Jacob, K.G Srinivasagan, “An image Indexing and Retrieval Algorithm Using Local Tetra Texture Features", International Journal of Advanced research in Computer Science and Software Engineering, Vol. 3, Issu.3, 2013

[34] M. Heikkilä and M. Pietikainen, "A texture-based method for modelingthe background and detecting moving objects," IEEE Trans. PatternAnal. Machine Intell., vol. 28, no. 4, pp. 657-662, Apr. 2006.

[35] M. Heikkilä, M. Pietikainen, and C. Schmid, "Description of interest regions with local binary patterns," Pattern Recognit., vol. 42, pp.425-436, 2009.

[36] L. Setia, A. Teynor, A. Halawani, and H. Burkhardt, "Image classification using cluster co-occurrence matrices of local relational features," in Proc. 8th ACM Int. Workshop on Multimedia Information Retrieval,2006, pp. 173-182.

[37] M.Joseph Prakash, Saka.Kezia, Dr.I.Santi Prabha, Dr.V.Vijaya Kumar, "A New Approach for Texture Segmentation using Gray Level Textons", International Journal of Signal and Image Processing, Vol. 6, No. 3, 81-89, June 2013

[38] K. Valkealahti and E. Oja, "Reduced multidimensional co-occurrence histograms in texture classification," IEEE Trans. Pattern Anal. Machine Intell., vol. 20, no. 1, pp. 90-94, Jan. 1998.

[39] T. Ojala, K. Valkealahti, E. Oja, and M. Pietikäinen, "Texture discriminationwith multidimensional distributions of signed gray-level differences," Pattern Recognit., vol. 34, no. 3, pp. 727-739, 2001

[40] T. Leung and J. Malik, "Representing and recognizing the visual appearance of materials using three-dimensional textons," Int. J. Comput.Vision, vol. 43, no. 1, pp. 29-44, 2001.

[41] Serratosa and A. Sanfeliu, "Signatures versus histograms: Definitions,distances and algorithms," Pattern Recognit., vol. 39, no. 5, pp.921-934, 2006.

[42] B.Eswara Reddy, P.Chandra Sekhar Reddy, V.Vijaya Kumar, "Texton based shape features on local binary pattern for age classification", International journal of image, graphics and signal processing (IJIGSP), Vol. 7, No.4,pp.54-60, July-2012, ISSN: 2074-9074.

[43] PS Kumar, VV P. Kiran Kumar Reddy, ”Age classification based on features extracted from third order neighbourhood Local Binary Pattern", ICTACT journal on image and video processing 5(2),926-931 
[44] T. Ahonen and M. Pietikaïnen, "Image description using joint distribution of filter bank responses," Pattern Recognit. Lett., vol. 30, pp.368-376, 2009.

[45] X. Tan and B. Triggs, "Enhanced local texture feature sets for face recognition under difficult lighting conditions", IEEE Trans. Image. Process., Vol. 19, No. 6, pp: $1635-1650,2010$

[46] Coggins J.M. and Jain A.K., "A spatial filtering approach to texture analysis," Pattern Recogninon Letters, no.3, pp.195-203, 1985.

[47] P. Brodatz, Textures: "A Photographic Album for Artists and Designers ".New York, NY, USA: Dover, 1999.

[48] http://vismod.media.mit.edu/pub/VisTex/VisTex.tar.gz

[49] Abdulrahman Al-Janobi, "Performance evaluation of cross-diagonal texture matrix method of texture analysis", Pattern Recognition 34 (2001) 171 \}, 180.

[50] L. Wang, D.C. He, "A new statistical approach for texture analysis", Photogrammetric Engng. Remote Sens. 56 (1) (1990) 61-66

\section{Author's Profiles}

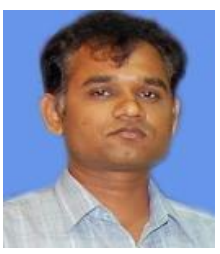

M. Srinivasa Rao received the B.Tech Computer Science \& Engineering from Nagarjuna University in $1998 . \mathrm{He}$ completed M.Tech in Software Engineering from JNT University, Masab Tank, and Hyderabad, India in 2001. He is having nearly 15 years of teaching and industrial experience. He is currently working as Asso ciate Professor, Dept of C.S.E, Sri Vasavi Institute of Engineering \& Technology, pedana, Andhrapradesh, India. He is pursuing his Ph.D. from JNT University, Kakinada in Computer Science \& Engineering under the guidance of Dr. V. Vijaya Kumar. He is a life member of ISTE and CSI. He published 5 papers in various conferences and journals.

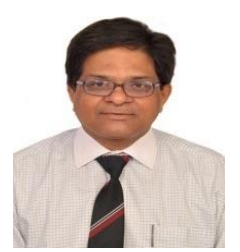

Dr. V. Vijaya Kumar is working as Dean in Dept. of CSE \& IT, chairman Board of studies for CSE and IT and Director Centre for Advanced Computational Research (CACR) at Anurag Group of Institutions, (AGOI) (Autonomous), Hyderabad. He received integrated M.S.Engg, in CSE from USSR in 1989. He received his Ph.D. degree in Computer
Science from Jawaharlal Nehru Technological University (JNTU), Hyderabad, India in 1998 and guided 28 research scholars for Ph.D. He acted as principle investigator for various R\&D projects. He has served JNT University for 13 years as Assistant Professor, Associate Professor and Professor. He has received best researcher and best teacher award from JNT University, Kakinada, India. His research interests include big data, image analytics, image retrieval, image processing, pattern recognition, digital water marking and cloud computing. At present he is also acting as BoS member for JNTUH and various other institutions. He is the life member of CSI, ISCA, ISTE, IE (I), IETE, ACCS, CRSI, IRS and REDCROSS. He published more than 100 research publications till now in various national, international journals and conferences. He has delivered key note addresses at various international conferences.

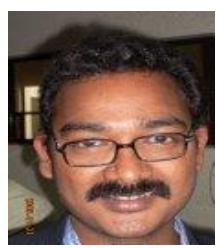

Dr. MHM Krishna Prasad is currently an Professor of the Department of Computer Science and Engineering, University College of Engineering, Kakinada (Autonomous), JNTUK, Andhra Pradesh. He did his B.E. from Osmania University, Hyderabad, M.Tech. and Ph.D. Computer Science and Engineering from JNTU, Hyderabad. He successfully completed a two year MIUR fellowship at University of Udine, Udine, Italy. He has about 50+ research papers in various International Journals and Conferences, and attended many national and international conferences in India and abroad. $\mathrm{He}$ is a member of Association for Computing Machinery (ACM), ISTE and IAENG (Germany) is an active member of the board of reviewers in various International Journals and Conferences. His research interests include data mining, Big Data Analytics and High Performance Computing.

How to cite this paper: M. Srinivasa Rao, V.Vijaya Kumar, Mhm Krishna Prasad,"Texture Classification based on First Order Local Ternary Direction Patterns", International Journal of Image, Graphics and Signal Processing(IJIGSP), Vol.9, No.2, pp.46-54, 2017.DOI: 10.5815/ijigsp.2017.02.06 